\title{
RESOURCE CONSUMPTION ACCOUNTING RCA: METODOLOGIA ALTERNATIVA PARA GESTÃO DE CUSTOS
}

\author{
RESOURCE CONSUMPTION ACCOUNTING RCA: ALTERNATIVE APPROACH FOR COST \\ MANAGEMENT
}

Wendy Beatriz Witt Haddad Carraro

Professora Adjunta da Universidade Federal do Rio Grande do Sul do Departamento de Ciências Contábeis e Atuariais, e do Programa de Pós Graduação em Contabilidade (PPGCONT) da UFRGS. Pesquisadora do Instituto de Avaliação de Tecnologia em Saúde - IATS. Integrante e vice-coordenadora do Núcleo de Empreendedorismo da UFRGS

Porto Alegre, RS, Brasil

E-mail: wendy.carraro@ufrgs.br

\section{RESUMO}

A gestão estratégica de custos representa um papel cada vez mais importante nas organizações. Seu principal objetivo é atingir uma vantagem competitiva através da redução dos custos, favorecendo, consequentemente, a formação de um menor preço de mercado, ou seja, mais competitivo e que demandará maior produção gerando mais receita e lucros, culminando no objetivo principal de uma organização no contexto globalizado. O objetivo deste artigo, é explorar a conceituação, operacionalização, as aplicações e limitações da metodologia do Resource Consumption Accounting (RCA) ou Contabilidade de Consumo de Recursos, que constitui uma nova abordagem na gestão estratégica de custos e que fornece aos gestores informações otimizadas sobre a organização. Objetiva, também, apresentar os níveis de maturidade na aplicação da gestão de custos, onde se destaca a trajetória para alcançar a utilização da metodologia RCA. Buscou-se através desta pesquisa apresentar uma metodologia para aplicação do RCA, que é a análise dos níveis de maturidade dos custos. O nível 11 é a abordagem do Resource Consumption Accounting. O RCA realça os recursos e as características de seu custo como o ponto de partida para todos os fluxos de custos através do modelo. Essencialmente o RCA combina a aprendizagem, aplicabilidade e princípios para tomada de decisões, permitindo uma gestão adequada e eficiente do negócio.

Palavras-chave: Gestão Estratégica de Custos. Metodologia de Gestão de Custos. Contabilidade de Consumo de Recursos. Níveis de Maturidade de Custos.

\section{ABSTRACT}

The strategic cost management is an increasingly important role in organizations. Its main objective is to achieve a competitive advantage by reducing costs, favoring thus the formation of a lower market price, ie, more competitive and that increased production will require generating more revenue and profits, culminating in the main objective of an organization in global context. The Strategic Cost Management is used in planning, control, and especially in decisionmaking. This article, in particular, is exploring the concept, operation, applications and limitations of the methodology of Resource Consumption Accounting $(R C A)$, which is a new approach to strategic cost management and provides managers optimized information about the organization. It also aims to present levels of maturity in the application of cost management, which includes the path to achieve using the RCA method. Was sought through this research provide a methodology for implementation of the RCA, which is the analysis of the maturity levels of costs. The level 11 is the approach of Resource Consumption Accounting. The RCA enhances the resources and the characteristics of its cost as the starting point for all cost flows through the model. Essentially, the RCA combines learning, applicability and principles for decision-making, allowing a suitable and efficient business management.

Keywords: Cost Strategic Management. Cost Masnagement Approach. Resources Consumption Accounting. Cost Maturity Levels. 


\section{INTRODUÇÃO}

Cada vez mais a sociedade vem se transformado, vivendo um novo cenário de ordem social e econômica, e consequentemente, passando a exigir mais das organizações. Esta exigência corresponde não apenas a conquistar um lugar ou espaço, mas também o de se manter e sobreviver no mercado. É necessário que as organizações desenvolvam métodos eficientes que possibilitem uma melhor gestão dos negócios, frente a esta competitividade, cada vez mais crescente, estabelecida pela globalização. Para alcançar um diferencial, é preciso que a organização tenha como foco uma gestão adequada, almejando permanecer no mercado.

Segundo Pompermayer e Lima (2002) o lucro deixou de ser atributo da receita e das vendas, e passou a ser função resultante dos custos incorridos, isso significa que almejar lucro é conter custos, e que, em qualquer conjuntura, é sempre possível conter custos. Neste sentido, este passa a ser um diferencial das empresas, aquela que conseguir desenvolver uma boa gestão de custos, terá seus lucros maximizados e, como resultado, assegurada sua permanência no mercado.

A Gestão Estratégica de Custos passa a representar um papel cada vez mais importante nas organizações. Segundo Hansen e Mowen (2001) a Gestão Estratégica de Custos corresponde ao uso de dados de custos que permitem desenvolver e identificar as estratégias que são superiores nas práticas da organização e que, de alguma forma, produzirão uma vantagem competitiva sustentável. Essa definição indica que o principal objetivo da Gestão Estratégica de Custos é atingir uma vantagem competitiva através da redução dos custos, favorecendo, consequentemente, a formação de um menor preço de mercado, ou seja, mais competitivo, que demandará maior produção, gerando mais receita e lucros, culminando no objetivo principal de uma organização no contexto globalizado.

A gestão de custos possuiu uma característica ampla, pois abrange a organização e suas relações estabelecidas ao longo da cadeia de valor, uma vez que possui um compromisso com a racionalidade econômica das decisões e com a geração de informações necessárias para avaliação de resultado, bem como para o processo de tomada de decisão.

Um sistema eficiente de custos possibilita à empresa: (a) Ter dados para o estabelecimento de padrões, orçamentos e outras formas de previsão; (b) Acompanhar os gastos efetivamente ocorridos e compará-los com os valores anteriormente definidos; (c) Estabelecer preços de vendas compatíveis com o mercado em que atua, (d) Conhecer a lucratividade de cada produto; (e) Decidir sobre cortes de produtos; (f) Reduzir custos; (g) Determinar o ponto de equilíbrio e; (h) avaliar o desempenho (CREPALDI, 2009). Dessa forma a Gestão Estratégica de Custos passa a ser utilizada no planejamento, no controle e, principalmente, na tomada de decisões.

Há diferentes abordagens metodológicas em relação à gestão dos custos nas organizações. O objetivo deste artigo, em particular, é explorar a conceituação, operacionalização, as aplicações e limitações da metodologia do Resource Consumption Accounting (RCA) ou Contabilidade de Consumo de Recursos, que constitui uma nova abordagem da gestão estratégica de custos e que fornece aos gestores informações otimizadas sobre a organização. Objetiva, também, apresentar os níveis de maturidade na aplicação da gestão de custos, onde se destaca a da trajetória para alcançar a utilização da metodologia RCA. Essencialmente, o RCA combina aprendizagem, aplicabilidade e princípios para tomada de decisões, 
permitindo uma adequada e eficiente gestão do negócio. A abordagem foi concebida em meados do ano 2000, principalmente como uma fusão da metodologia de gestão de custos alemã Grenzplankostenrechnung (GPK) e das práticas do Activity-Based Costing (ABC). O RCA então permaneceu durante sete anos em incubação para ser validado e ter a adequação de seus princípios, conceitos e métodos através de estudos de casos práticos e de pesquisa.

A metodologia utilizada na execução deste trabalho é a de pesquisa bibliográfica, buscando na literatura existente informações disponíveis e relevantes sobre a metodologia RCA. Segundo Gil (1999), a pesquisa bibliográfica é desenvolvida com material já elaborado sobre o tema. Segundo Marconi e Lakatos (2002) a pesquisa bibliográfica permite buscar uma solução ao problema pesquisa, bem como identificar novas problemáticas sobre o tema. A abordagem do estudo é qualitativa. Segundo Richardson (1999) os estudos que pregam uma metodologia qualitativa permitem a descrição da complexidade de determinado problema, bem como analisar a interação de certas variáveis, compreendendo e classificando os processos dinâmicos vivenciados, possibilitando o entendimento das particularidades dos comportamentos.

O presente estudo está estruturado em cinco seções, inicia com esta introdução. Em seguida, apresenta as descrições das metodologias que deram origem ao RCA: Activity-Based Costing (ABC) e Grenzplankostenrechnung (GPK), apresentando suas aplicações, vantagens e desvantagens. Na sequência, apresenta a conceituação, aplicação, vantagens e limitações da abordagem RCA, descrevendo situações que indicaram perfomance e resultados positivos de sua aplicação. Após, descreve os níveis de maturidade na aplicação da gestão de custos, onde se destaca a importância e relevância da trajetória para alcançar a utilização da metodologia RCA. Para finalizar, apresenta as considerações finais do trabalho.

\section{ORIGENS DO RESOURCE CONSUMPTION ACCOUNTING - RCA}

A Contabilidade do Consumo de Recursos (RCA) é um sistema de gerenciamento do custo bastante abrangente e plenamente integrado. É uma abordagem do gerenciamento de custo que influencia as últimas décadas no que diz respeito a esse assunto na Europa e nos Estados Unidos. O RCA combina o confiante sistema alemão de gerenciamento de custo (GKP) com o Activity-Based Costing (ABC). Essa fusão dos dois mundos fornece uma abordagem completa e compreensível do gerenciamento da contabilidade. Nesta seção serão apresentadas as principais características das duas metodologias que deram origem ao RCA.

\section{Activity-Based Costing - ABC}

Conhecida como ABC (Activity-based Costing), esta ferramenta de gestão de custos busca a redução, de forma bastante sensível, das distorções provocadas pelos métodos de rateio arbitrário dos custos indiretos, embora esse método possa também ser aplicado aos custos diretos, principalmente à mão-de-obra direta, apesar de não trazer diferenças significativas, se comparado aos métodos tradicionais de custeio (MARTINS, 2003). O ABC busca o entendimento da formação de todos os custos necessários à obtenção de determinado produto ou serviço, bem como determinar a sua respectiva alocação, com o objetivo de alcançar uma avaliação de custos mais adequada. 
Segundo Ching (2001), o sistema ABC apresenta a forma como uma empresa emprega tempo e recursos para atingir determinados objetivos. $O$ autor destaca que, mesmo antes de se focar propriamente $o$ que é o $A B C$, é preciso conhecer o que é atividade. Chama atenção de que uma atividade pode caracterizarse como o agente consumidor de recursos para produzir um output, seja ele um produto ou serviço. Para Santos, Schmidt e Leal (2009) o critério ABC é uma metodologia de custeio que procura reduzir sensivelmente as distorções provocadas pelo rateio arbitrário dos custos indiretos.

A busca pelo baixo custo, sem afetar a estrutura e a qualidade dos produtos e serviços, deve ser o foco constante nas organizações que visam sua excelência. Nesse sentido, o $A B C$, representa uma excelente ferramenta no gerenciamento de custos, com várias aplicações dentro das organizações, permitindo análises detalhadas e compreensivas de custos de qualquer natureza, sempre sob a ótica das atividades desenvolvidas e a respectiva contribuição, ou seja, o quanto geram de agregação de valor para o negócio.

$O$ ponto inicial para gerenciar atividades é entender os recursos, ponto de convergência com a metodologia RCA, bem como o equilíbrio entre o fornecimento de recursos à disposição da empresa e a demanda desses mesmos recursos exigidos pelas atividades. $O A B C$ permite balancear adequadamente a demanda e o fornecimento de recursos disponíveis por parte da empresa e mostra detalhadamente os recursos consumidos pelas atividades através de um mapa.

Kaplan e Anderson (2007) descrevem que o método ABC é estruturado em duas etapas distintas: na primeira os recursos consumidos são alocados às atividades; na segunda, os custos das atividades são alocados aos objetos de custo, sejam estes produtos, serviços ou clientes. O método baseia-se, portanto, no princípio de que são as atividades desenvolvidas na empresa que causam os custos, ao consumir os recursos, e de que são os objetos de custos que consomem as atividades. Qualquer mudança no número de atividades ou alteração no modo como as atividades são realizadas, faz com que os custos aumentem ou diminuam.

Em estudo recente, Souza, Silva e Pilz (2010) apontaram que algumas práticas foram assinaladas como não utilizadas e sem qualquer sinalização de provável uso futuro. Destacando entre elas o $A B C$ e a gestão baseada em atividades (ABM). Em relação ao uso das atividades como base de práticas específicas, seja no custeio $(A B C)$ ou na gestão ( $A B M)$, há uma forte alegação da desfavorável relação custo $x$ benefício, um argumento bastante presente quando são investigadas tais práticas. Segundo os autores, a não identificação objetiva dos possíveis benefícios ainda está bloqueando a adoção do ABC.

Schmidt, Santos e Leal (2009) destacam que para muito pesquisadores, a forte resistência à adoção e à sustentação da metodologia $A B C$ é racional e justificada, pois, para os autores, tal sistema se caracterizou pelo alto custo de desenvolvimento, pela complexidade de manutenção e pela dificuldade de modificação. Também destacam a questão da dúvida com relação à exatidão das alocações de custos, baseadas em estimativas individuais subjetivas sobre os percentuais dos seus respectivos tempos dedicados a diferentes atividades. Concluem que, quase todos os sistemas $A B C$, calculam as taxas dos direcionadores de custo com base na presunção de que os recursos atuam a plena capacidade. 


\section{Grenzplankostenrechnung - GPK}

O sistema GPK iniciou na área de fabricação e depois se espalhou para aplicação também em organizações prestadoras de serviços. As primeiras utilizações estavam focadas somente no custo direto do produto, criando a impressão de que era apenas uma metodologia de custeio marginal. Os elementos essenciais do sistema GPK, apresentados por Plaut (1953, apud Sharman e Vikas, 2004), consideram que os centros de custos devam ser de total responsabilidade de uma gerência, representando os efeitos e custos homogêneos, sendo econômicos no design (não muito complexos), indicando o custo dos recursos empregados, como, por exemplo, quando um recurso se equipara às máquinas, pessoas, edifícios, etc. Em relação à fragmentação dos custos fixos e proporcionais, essa deve ser feita para cada centro de custo por conta/atividade, aplicando condutores de recursos para distribuir os custos, empregando limitações de capacidade/utilização e considerando como os custos se comportam com as alterações nos níveis de produção.

As atividades e os condutores devem ser aplicados de modo que cada centro de custo tenha pelo menos uma média de produção, horas/máquina, por exemplo, de forma a garantir que a relação entre a média de produção e o pool do custo proporcional seja linear, e que a relação entre a média de produção e o objeto (produto) seja igualmente linear. O planejamento de custo analítico deve ser um componente integral do orçamento e planejamento operacional, sendo realizado para cada centro de custo, o que deve refletir a demanda sob condições efetivas, eficientes e econômicas (expectativas realistas versus "último ano + X\%").

A alocação correta dos serviços de custo interno deve refletir as verdadeiras condições da oferta e da demanda, aplicar o "pull" da lógica, que demonstra o fluxo de recursos para a produção ou objeto de custo como custo do "pull" de produtos. Garantindo que a caracterização do custo como proporcional ou fixo seja mantida, assim como a cascata de custos através do sistema. O uso dos padrões deve ser estável durante o período de planejamento, bem como ser a base e fornecer a compreensão das variâncias (preço, utilização). Com base nestas características do GPK, Plaut (1953) apud Sharman e Vikas (2004) e seus associados, passaram a implementar o GPK em centenas de organizações, muitas das quais ainda o estão utilizando, mesmo após décadas de sua implementação.

Entretanto, há alguns aspectos críticos quando da implantação inicial da utilização do sistema GPK que devem ser destacados: (a) A metodologia estava direcionada a uma ênfase na análise de custos diretos ou de custos que variam em proporção à produção de atividade (o que os alemães descrevem como "proporcional"). (b) O controle no nível de centro de custos era bastante crítico, apresentando cálculos manuais significativamente extensivos, uma vez que não havia muita utilização de computadores nas primeiras utilizações do sistema. (c) O custeio marginal foi utilizado para decidir quais produtos deveriam ser eliminados e em quais produtos concentrar todos os esforços de marketing e de vendas. (d) A margem de contribuição foi calculada para os produtos e clientes e foi utilizada para influenciar os esforços da equipe de vendas. $E(e)$ a falta de um padrão de software criou uma dependência por processamento de dados nos departamentos de Tl, que carregou junto problemas associados com os longos ciclos de desenvolvimento.

Porém, como as capacidades da informática evoluíram, Plaut (1953, apud Sharman e Vikas, 2004) e seus associados, trabalharam na consultoria com cada cliente para produzir seu próprio software customizado e autônomo, com o objetivo de facilitar a captura de dados e calcular os resultados. O processo, entretanto, 
foi ineficiente, complicado e repetitivo. Após algum tempo, as empresas começaram a trocar informações umas com as outras, criando grupos de usuários para estabelecer as melhores práticas. O que, por sua vez, resultou em empresas de partilha de software, criando diversos problemas devido à falta de práticas disciplinadas de introdução do produto e aplicações inconsistentes. Tais problemas, eventualmente, levaram Plaut (1953) apud Sharman e Vikas (2004) a desenvolver um sistema padrão e autônomo do custeio de gerenciamento.

Para manter o ritmo com a mudança de tecnologia e, para tentar tirar proveito dos dados disponibilizados pelas soluções integradas, o referido autor acabou por ser levado à abordagem do principal fornecedor alemão a nível mundial de sistemas integrados, SAP, em meados da década de 80 para que eles produzissem a solução de seus sistemas. Foi exatamente isso que o SAP fez, e Plaut (1953, apud Sharman e Vikas, 2004) saiu do negócio de software. Ele ainda trabalhou com clientes SAP para ajudá-los a implementar o sistema GPK corretamente. A metodologia foi incorporada no módulo "Controle", cujos dados operacionais foram retirados de outros módulos, como, por exemplo, o faturamento ou a gerência de produção; e os dados financeiros foram retirados do módulo de informações financeiras. O novo sistema proporcionou uma integração e padronização, eliminando, assim, os problemas associados com sistemas autônomos.

Em um encontro realizado em Harvard em 1989, representantes do SAP discutiram métodos e aplicações de custeio alemão, comparando-os com custeio baseado em atividades que estava começando a emergir nos EUA. O software ABC foi desde o seu início nos EUA (assim como os sistemas autônomos e as soluções personalizadas na Alemanha) desenvolvido pelas organizações que lutavam para vender ou implementar o ABC. Consequentemente, a maioria das organizações americanas que haviam investido milhões de dólares em softwares do SAP falhou ao tentar tirar vantagem da capacidade total do módulo de controle do SAP CO. A decisão dessas organizações de não utilizar o SAP CO parecia estranha, dada a quantidade de dinheiro que gastaram para adquirir e implementar o software SAP. Possivelmente é porque a abordagem do SAP era diferente do ABC, que estava se tornando popular nos EUA, e os profissionais, acadêmicos e consultores não o entenderam. Felizmente, o SAP tem evoluído seu sistema constantemente para incorporar as boas práticas e os padrões do sistema ABC.

\section{Resource Consumption Accounting - RCA}

O Resource Consumption Accounting (RCA), ou Contabilidade de Consumo de Recursos, é um abrangente sistema de gestão totalmente integrado de custos, constitui uma nova abordagem da contabilidade e fornece aos gestores informações otimizadas sobre a organização. Essencialmente, o RCA combina a aprendizagem, aplicabilidade e os princípios para tomada de decisões, permitindo uma adequada e eficiente gestão do negócio.

Segundo Merwe e Keyes (2002) a abordagem foi concebida em meados do ano 2000, principalmente como uma fusão da metodologia de gestão de custos alemã Grenzplankostenrechnung (GPK) e das práticas do Activity-Based Costing (ABC). O RCA passou sete anos em incubação para ser validado e ter definida a adequação de seus princípios, conceitos e métodos através de estudos de casos práticos e de pesquisa. Essa fusão, do melhor de dois mundos, prevê uma abordagem integrada e abrangente para a contabilidade 
de gestão. Três pilares são essenciais para o RCA: (1) A visão dos recursos; (2) A visão da natureza do custo; e, (3) uma abordagem baseada na quantidade da modelagem do custo.

Segundo o RCA Institute (2011), em julho de 2009, o RCA foi reconhecido pela International Federation of Accounting's International Good Practice Guide: Evaluating and Improving Costing in Organization, bem como pelo Information Paper to the IGPG, Evaluating the Costing Journey: A Costing Levels Continuum Maturity Model, como uma das mais sofisticadas abordagens de custeio.

Segundo Clinton e Webber (2004) o Resource Consumption Accounting (RCA) ou Contabilidade de Consumo de Recursos é um novo método de custos que fornece resultados mais exatos e informações mais detalhadas, quando comparado aos métodos tradicionais. Segundo os autores, os sistemas tradicionais, normalmente, fornecem dados distorcidos em relação aos custos e, simplesmente, não disponibilizam o detalhamento registrado pelo RCA.

Quando um administrador toma uma decisão específica, como por exemplo, terceirizar ou fabricar um produto, ele deve estar confiante de que o RCA fornecerá os dados exatos para auxiliá-lo na decisão. Devido à exatidão e ao detalhamento das informações que o RCA fornece, os tomadores de decisão têm os dados que precisam para alinharem suas ações para a realização das estratégias da empresa. Baseado nos princípios de gerenciamento de custos conforme o método de custeio Grenzplankostenrechnung (GPK), o RCA funciona bem com sistemas de planejamento de recursos das empresas (Enterprise Resource Planning (ERP), pois obtém informações desde o nível primário (como a manufatura) para apurar os custos com exatidão.

O RCA tem como foco os recursos, onde é verificada, de forma detalhada, a quantidade de recursos consumida, considerando que a natureza dos custos alterará. O RCA apresenta três princípios fundamentais, o primeiro diz respeito ao reconhecimento das relações múltiplas e recíprocas; o segundo é em relação à inclusão de todos os custos imputáveis na atribuição de custos; e por último, mas não menos importante, a questão do fornecimento de uma estrutura para gestão da capacidade da organização.

Segundo Merwe e Keyes (2002) o princípio do RCA de reconhecer as mudanças naturais e potencias dos custos concentra-se em dois aspectos, primeiro em determinar a natureza inata de um item do custo na aquisição baseado na decisão do administrador ou planejar prevendo como o recurso será consumido, e segundo, em mudar os custos que foram inicialmente proporcionais por natureza para fixos baseados em padrões de consumo.

Segundo o Good Practice Guidance (2009) o RCA utiliza três elementos fundamentais na modelagem operacional, os quais the permitem estabelecer uma base muito diferente para seu modelo de custos em comparação às abordagens tradicionais de custeio:

1. A visão de recursos, onde os recursos e seus custos são considerados nesta abordagem como fundamentais para a modelagem adequada dos custos e apoio à decisão. Os custos e receitas da organização são funções dos recursos que os produzem.

2. A modelagem baseada na quantidade, onde o modelo de custo total é calculado utilizando quantidades operacionais. Os dados operacionais representam o fundamento da criação de valor, e o principal indicador de resultados econômicos.

3. O comportamento dos custos, onde o valor adicionado é um elemento importante para o modelo baseado na quantidade do comportamento dos custos, ou seja, ele é determinado pelo 
comportamento das quantidades de recursos subjacentes, como eles são aplicados às operações de criação de valor dentro de uma organização.

A partir desses elementos, destaca-se o potencial do RCA como instrumento válido para o processo de planejamento e controle em uma empresa. A partir de dados do projeto piloto de utilização do RCA, Clinton e Webber (2004) apontam que os resultados da metodologia fornecem exatidão no aumento do custo do produto e facilitam a capacidade de gerenciamento, entre outras vantagens. A Quadro 1 apresenta os benefícios significativos do RCA em comparação com sistemas tradicionais de Gerenciamento de Custos, levantados pelos autores.

Quadro 1 - Benefícios Importantes do RCA com Relação a Sistemas Tradicionais de Gerenciamento de Custos

\begin{tabular}{|c|c|}
\hline RCA & Tradicional \\
\hline $\begin{array}{l}\text { Atribui os custos da capacidade excedente/ociosa à pessoa ou } \\
\text { ao nível responsável por influenciar o recurso, mas não aloca } \\
\text { ao produto. }\end{array}$ & $\begin{array}{l}\text { A capacidade excedente/ociosa não é identificada e por isso não } \\
\text { pode ser associada à pessoa ou ao nível responsável e } \\
\text { frequentemente é alocada aos produtos. }\end{array}$ \\
\hline $\begin{array}{l}\text { Análise da capacidade das instalações através da utilização da } \\
\text { capacidade teórica das taxas de custos e tornando a } \\
\text { capacidade excedente/ociosa visível aos administradores. }\end{array}$ & $\begin{array}{l}\text { Oculta a análise da capacidade através da utilização máxima de } \\
\text { orçamento para taxa de custos e não contabiliza a capacidade } \\
\text { excedente/ociosa. }\end{array}$ \\
\hline $\begin{array}{l}\text { Utiliza a substituição do custo de depreciação para fornecer } \\
\text { informações que sustentem a utilização dos custos internos. }\end{array}$ & $\begin{array}{l}\text { Utiliza a depreciação determinada por um sistema de relatórios } \\
\text { externo que geralmente não pondera a realidade econômica. }\end{array}$ \\
\hline $\begin{array}{l}\text { Considera o custo dos recursos consumidos como custo dos } \\
\text { objetos através da utilização de não-monetária, relações de } \\
\text { consumo claramente quantificadas baseadas na causalidade. }\end{array}$ & $\begin{array}{l}\text { Considera o custo dos recursos fornecidos no custo dos objetos } \\
\text { através da difusão de todos os custos incorridos durante a produção } \\
\text { de bens acabados. }\end{array}$ \\
\hline $\begin{array}{l}\text { Identifica e atribui os custos como fixos ou variáveis por } \\
\text { natureza (proporcionalmente) no nível de recursos, } \\
\text { especificando devidamente a natureza dos custos. }\end{array}$ & $\begin{array}{l}\text { Identifica e atribui os custos como fixos ou variáveis por natureza } \\
\text { no nível do produto, ocultando o verdadeiro modelo do custo de } \\
\text { consumo. }\end{array}$ \\
\hline $\begin{array}{l}\text { Reconhece que os custos proporcionais por natureza podem } \\
\text { ser consumidos de uma forma fixa e fornece tratamento } \\
\text { necessário. }\end{array}$ & $\begin{array}{l}\text { Não fornece reconhecimento do padrão de consumo de custos no } \\
\text { nível de recursos. }\end{array}$ \\
\hline $\begin{array}{l}\text { Fornece aos tomadores de decisão a capacidade para localizar } \\
\text { as informações dos grupos de custos em qualquer nível - do } \\
\text { nível de recursos até o nível da organização. }\end{array}$ & $\begin{array}{l}\text { Os grupos de custos de um setor ou nível de produto com pouca ou } \\
\text { nenhuma provisão para localizar ou acessar os custos de níveis } \\
\text { mais baixos. }\end{array}$ \\
\hline $\begin{array}{l}\text { Facilita o gerenciamento das operações com informações não } \\
\text { financeiras atuais e quantificadas para comparar com as } \\
\text { quantidades planejadas ou padronizadas. }\end{array}$ & $\begin{array}{l}\text { Informações não financeiras frequentemente são escassas e } \\
\text { indisponíveis, uma vez que os recursos estão alocados com base } \\
\text { no percentual de relacionamento sem localizar o consumo da } \\
\text { quantidade de recurso. }\end{array}$ \\
\hline
\end{tabular}

Fonte: Adaptado de Clinton e Webber (2004).

Para fins de ilustração, destaca-se um dos cases de maior relevância em relação aos resultados gerados a partir da implementação do RCA. Segundo Clinton e Webber (2004), a empresa Cloplay utilizou o RCA para criar 23 pools de recursos divididos em duas categorias: suporte geral e setores de produção. A utilização de 23 pools ao contrário de oito suportes e seis setores de produção tornava possível determinar o custo de acordo com o pool de recursos. Quanto mais pools de recursos, mais informações detalhadas, o que tornava os dados precisos quando utilizados para tomadas de decisão.

Essa abordagem possibilitou maior homogeneidade conquistada pela Cloplay através da utilização de setores que continham uma diversidade de custos. O RCA exclui os custos fixos que não podem ser detectados com base na causalidade - a maioria desses custos era decorrente da capacidade ociosa. O RCA 
apresenta uma redução significativa no custo de conversão, quando comparado ao sistema de custos utilizado anteriormente pela empresa.

Ainda como complementação à aplicabilidade da metodologia, destacam-se Merwe e Keyes (2002) ao combinarem os princípios do RCA com orçamentação baseada em atividades tradicionais e planejamento de recursos baseado em atividades. Os autores propiciaram a possibilidade de fornecer previsões precisas da demanda de recursos e custos relacionados a recursos, bem como justificar adequadamente a relação capacidade planejada/ociosa excedente.

\section{MODELO CONTÍNUO DOS CUSTOS E NÍVEIS DE MATURIDADE}

O objetivo desta seção é apresentar o modelo contínuo de custos e os níveis de maturidade como ferramenta direcionadora para identificação e aplicação da metodologia RCA. O modelo fornece recursos complementares apresentados pelo Guia Internacional das Boas Práticas na Melhoria e Evolução do Custeio nas Organizações, permitindo da mesma forma que o International Federation of Accountants - IFAC atraia seus membros e integrantes ao desenvolvimento de futuras melhorias desse modelo. $\mathrm{O}$ modelo de maturidade de custo tem como objetivo auxiliar os profissionais da área de gestão de custos a julgar corretamente quando a capacidade de uma empresa apóia as análises e decisões do gerenciamento interno. O modelo também pode auxiliar as organizações a decidirem qual nível almejam para requisitos organizacionais previstos e para atender os requisitos empresariais e as decisões dos funcionários.

É uma ferramenta de auto-avaliação que pode ser utilizada por profissionais da área para discutirem com colegas a atual prática da organização e para ponderarem a ação apropriada, visando melhorar a análise e avaliação de desempenho, bem como para o planejamento e apoio de decisão. Esse diálogo possibilita uma melhor identificação da situação da empresa e a viabilidade de implementação dos diferentes métodos de custeio, especialmente, a identificação da empresa quanto à sua estrutura para aplicação do RCA, que é escopo deste trabalho. A figura 1 apresenta a trajetória de maturidade dos custos, sugerida pelo IFAC, e no decorrer desta seção serão apresentadas as características específicas de cada um dos níveis. 
Figura 1: Trajetória de Maturidade dos Custos

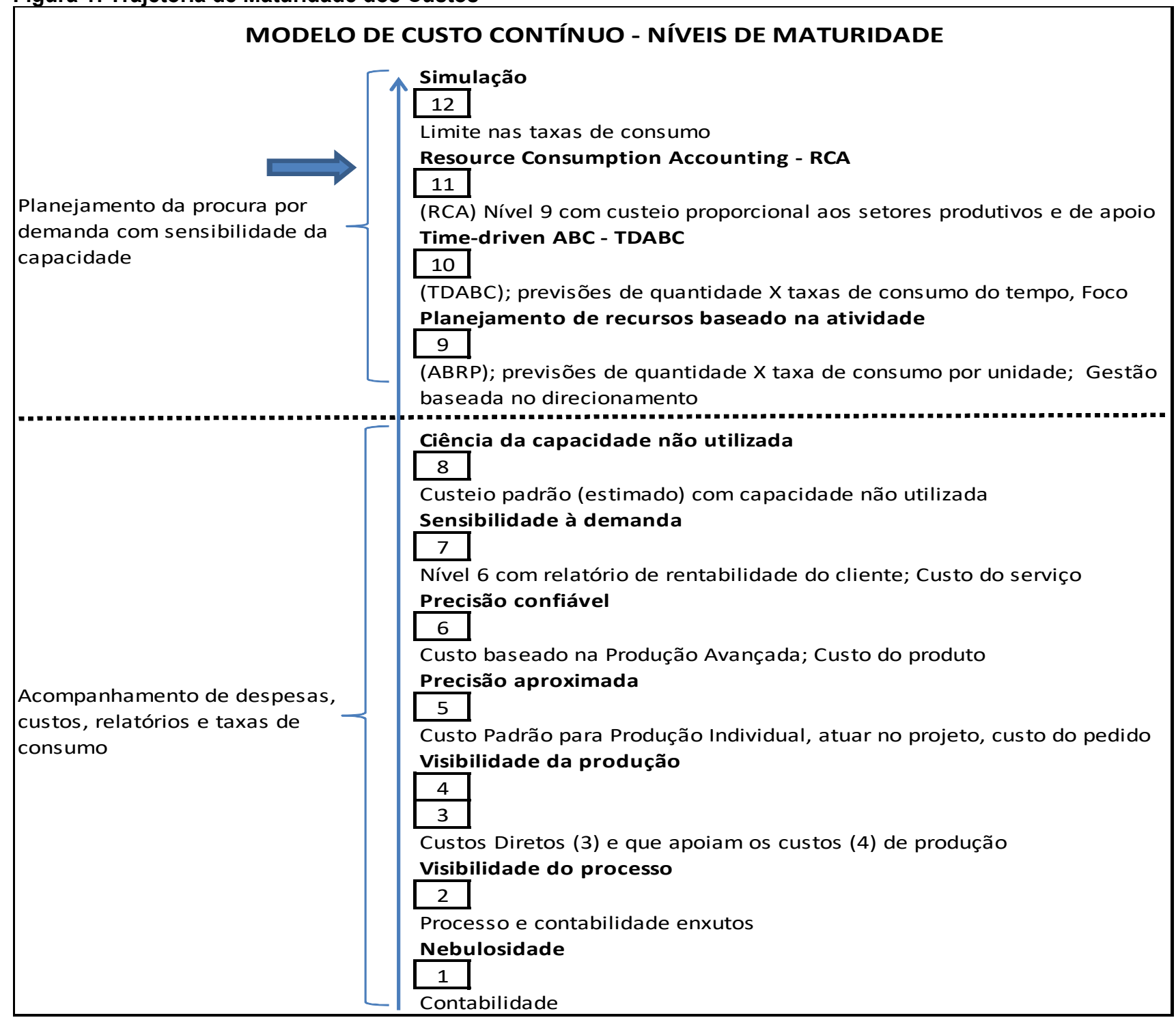

Fonte: Adaptada de IFAC (2011).

Embora a trajetória de maturidade seja representada por 12 níveis distintos, algumas organizações podem estar em níveis intermediários ou em vários níveis. A importância deste modelo de maturidade de custo é destacar que os modelos de custo e sistemas de apoio devem refletir a realidade subjacente da maneira como as organizações atuam, até onde a acessibilidade e a relevância permitirem e, dentro do contexto das decisões necessárias para o negócio.

O modelo de maturidade de custo também pode ajudar gestores a criarem uma regra geral para orientar os usuários, incluindo aconselhar, por exemplo, na seleção do método de custo, na medida e na classificação do custo. Cada novo nível da série é uma expansão do anterior, o que significa que os esforços para alcançar o nível anterior e seu design e suas capacidades de modelagem de custo podem ser mantidos, modificados ou removidos. Mais benefícios se acumulam enquanto a organização melhora suas capacidades. 


\section{Níveis básicos dos princípios da maturidade do custo}

Dois princípios básicos se aplicam a todos os níveis da série do custo. O primeiro está relacionado às capacidades de recurso (tipos e quantidades), para o resultado, produtos ou serviços, e para os consumidores finais (por exemplo, clientes) que consumem os recursos. Esse é o princípio básico que combina e equilibra as relações entre demanda e oferta. O segundo está relacionado à natureza do custo da maneira como os recursos são consumidos nessas relações. Ou seja, esse princípio reconhece que alguns insumos (e, consequentemente, seus custos) mudam com a alteração da demanda de resultados, enquanto outras entradas (e seus custos) permanecem constantes, indiferentes às demandas por resultado (até que uma próxima unidade distinta de recurso seja necessária para expandir ou contrair capacidade).

Cada nível técnico de custeio é distinguido pela eficácia com que a organização incorpora esses dois princípios - e também melhora seu desempenho. Melhores informações de custo auxiliam a organização a entender como a demanda está relacionada à oferta, e como o custo se comporta através das relações de consumo. Então o seu melhor será a visibilidade, análise, planejamento, orçamento e tomadas de decisão.

\section{Nível 1 - Nebulosidade (Blind)}

O nível 1 refere-se à contabilidade e aos relatórios financeiros, e contempla basicamente a escrituração contábil. Os centros de custo são poucos e agregados a um alto nível, como, por exemplo: operações, vendas, marketing e administração. Os custos não são calculados e nenhuma informação que permita uma percepção dos efeitos das mudanças incrementais, no nível de recursos e custo, por exemplo, é fornecida. Somente as despesas são capturadas e acumuladas.

\section{Nível 2 - Visibilidade do Processo (Process Visibility)}

O nível 2 conecta e reúne os centros de custos primários na sequência de tempo. Essa técnica de custeio, conhecida como "contabilidade excessiva", é utilizada para apoiar o método de melhoria do processo chamado de mapeamento de fluxo de valor. Um fluxo de valor inclui todas as atividades com valor agregado relacionadas ao fornecimento de produtos e serviços específicos aos clientes. Normalmente, as etapas do processo do fluxo de valor (por exemplo, atividades do trabalho) para as quais os custos são calculados, também são marcadas com atributos que denotam valor (valor agregado versus valor não agregado) ou a quantidade de importância.

\section{Níveis 3 e 4 - Visibilidade de Produção (Output Visibility)}

O nível 3 inclui os custos dos processos que consomem recursos nos custos das saídas (por exemplo, produtos).) Nesse nível, inicia o cálculo dos custos de diferentes grupos de saída (por exemplo, famílias de serviços básicos ou de produtos). Os custos calculados são restritos aos custos diretos de produção que podem ser diretamente associados aos grupos de produção (saída), tais como os custos diretos de material e mão-de-obra.

O nível 4 é o primeiro nível da Série de Custeio que "atribui" custos indiretos e compartilhados aos custos dos objetos. O custo total de um departamento de apoio indireto é atribuído e adicionado aos centros de custos diretos (do nível 3) utilizando um simples fator de alocação, como a quantidade de saídas 
produzidas pelo centro de custo direto. Esses custos compartilhados são adicionados ao custo direto de material e mão-de-obra que anteriormente eram atribuídas aos grupos de produção. As definições do comportamento dos custos nos sistemas dos níveis 3 e 4 são simplistas e limitados ao custo final dos objetos. A percepção sobre os efeitos ocasionados pelas mudanças incrementais na produção é, por esse motivo, limitada.

\section{Nível 5 - Precisão Aproximada (Approximate Accuracy)}

O nível 5 é subdividido em duas etapas. O nível 5 a tem detalhes (custos diretos) mais exatos e aloca (custos indiretos) para saídas e produtos individuais, contrastando com os custos agregados e agrupados das saídas nos níveis 3 e 4 . No Nível 5 , os centros de trabalho (máquina ou mesa de processamento) dentro de um centro de custo são controlados individualmente. Assim, os custos acumulados de cada centro de trabalho estão diretamente associados a uma única unidade de produção para produzir o resultado individual e seu custo.

As quantidades são provenientes de padrões predeterminados que muitas vezes refletem médias históricas, e são avaliados em termos financeiros com taxas padrão ou fixas (\$ por minuto) no sistema de custeio. No nível $5 \mathrm{a}$, o uso das quantidades operacionais resulta em custos razoavelmente exatos e métricas padrão, pois eles são exatos. No entanto, a alocação indireta e os custos partilhados levam à exatidão, pois são combinados com os custos diretos utilizando o fator de encaminhamento, violando assim, parcialmente o "princípio da causalidade".

O nível $5 b$ se aplica às organizações com condições especiais, onde o trabalho da organização e os processos geralmente não são repetitivos ou recorrentes, e sua finalidade normalmente é exclusiva para o usuário final. Exemplos dessas organizações seriam: um escritório de advocacia, uma empresa de consultoria gerencial ou um "make-to-order" do custo de fabricação baseado na atividade (ABC), onde o $A B C$ baseado no tempo estima dois aspectos: (a) a capacidade prática de recursos comprometidos e os seus custos e (b) a unidade de tempo para realização de atividades transacionais.

\section{Nível 6 - Precisão Confiável (Reliable Accuracy)}

O nível 6 aumenta substancialmente a precisão das saídas, dos produtos e dos custos padrão da linha de serviço, através do rastreamento das despesas indiretas até às saídas de uma forma que seja mais consistente com o princípio da causalidade. Esse rastreamento do custo contrasta com a alocação dos custos indiretos, cuja base está na média mais ampla, sem relação do consumo com o comportamento dos recursos indiretos e seus custos (utilizados do nível 3 ao 5). O nível 6 é o nível onde os princípios do custo baseado em atividades $(A B C)$ são primeiramente aplicados. Há três mini-níveis do $A B C$ - 6a a 6c. Em cada um, as despesas com recursos são "empurradas" (de cima para baixo) por meio de custos da atividade e, eventualmente, re-atribuídos ao custo final dos produtos.

Isso contrasta com o custo quantitativo da "pressão da demanda" quantitativa (de baixo para cima), que começa no nível 9. Nos níveis de 6 a 9, a maioria das despesas de contabilidade são atribuídas diretamente das atividades dos pools de custo. Esses gastos estão em unidades monetárias. Ainda não há uma conexão explícita dos recursos e das características do seu custo com recurso dedicado do custo do objeto. Isso resulta em informações incompletas dos recursos, como é evidente, a prática comum nesse nível 
de alocação de atividades para as atividades. O que gera uma qualidade de informação incremental que é semelhante à do nível 5 - de forma mais detalhada.

\section{Nível 7 - Sensibilidade à Demanda (Customer Demand Sensivite)}

No nível 7, os clientes tornam-se o custo final dos produtos na rede de atribuição de custo. Sua existência cria, por fim, a necessidade de recursos e de uma estrutura de custos para a organização. $O$ volume de produtos e serviços e seus mix são rastreados. Neste nível isso se realiza, pois são adicionados custos calculados atribuíveis aos canais e aos clientes que não estavam incluídos no nível 6 . Em contraste com produtos e custos de serviços padrão, estes custos são tipicamente referidos como "os custos para servir". O custo final dos produtos no nível 7 também incluí as receitas associadas (ou seja, preço $\mathrm{x}$ volume) de produtos e serviços precificados. Adicionando os preços, margens de contribuição para os lucros podem ser comparados entre os produtos, canais e clientes para análise e possíveis ações.

A principal diferença entre as organizações no nível 7 daquelas no nível 6 é que as que estão no nível 7 rastreiam custos de não-produtos e não-serviços padrão, tais como marketing, distribuição e custos de venda, para grupos (segmentos) e, posteriormente, para canais individuais e clientes. Eles não alocam aqueles custos aos produtos, porque não existem relações causais. Se os custos são alocados, então os custos dos produtos seriam ilusoriamente exagerados.

\section{Nível 8 - Ciência da Capacidade não Utilizada (Unused Capacity Aware)}

As organizações que planejam no nível 8 já alcançaram o nível 6 ou 7. As organizações no nível 8 determinam, geralmente por estimativa, a quantidade de cada recurso ocioso ou capacidade não utilizada ou tempo (se houver), e atribuem estes custos ao custo do objeto de negócio sustentável chamado de "capacidade não utilizada ou ociosa". A capacidade de fornecimento de recursos para o trabalho. Os clientes criam demanda para essa capacidade, dando origem à necessidade de produtos e serviços com canais para recebê-los. Os recursos são ou não utilizados.

A maioria dos recursos é utilizada, e esses são rastreados e identificados em todos os níveis. Mas incluindo uma capacidade ociosa de despesa de recursos nas designações do custo ocorre um exagero em todos os custos do processo/atividade, bem como os custos de produção. Eles também irão gerar um exagero nas taxas de custo utilizadas para projeções. Na medida em que informações marginais/incrementais estão sendo levadas em conta, o nível 8 é considerado melhor em relação aos níveis mais baixos. Nele, o custo dos recursos e características do comportamento dos custos começam a ser enfatizados, portanto, lançando as bases para uma melhor qualidade de informação marginal. Por sua vez, ao tomar decisões de otimização, dá apoio ao desenvolvimento de insights sobre o ganho incremental que são resultado das mudanças na produção. 
Nível 9 - Planejamento de Recursos Baseado na Atividade (Pull Activity-based Resource Planning)

As organizações que planejam ao nível 9 atingiram o nível 8, mas o nível 9a inicia o cálculo de seus modelos de trás para frente, para determinar o impacto esperado sobre os recursos a partir do pedido de um cliente ocasional, ou a partir de uma alteração no processo. Esse método usa o modelo da demanda quantitativa "puxar" em oposição à técnica alternativa de custeio para "empurrar" os custos (de cima para baixo), através do modelo. Para os sistemas $A B C$, o nível 9a representa o início do planejamento de recursos baseados em atividades (ABRP). Com o ABRP, as organizações alavancam as relações de recursos e de consumo de atividade para calcular a capacidade necessária de recursos.

Um avanço fundamental do nível 6 ao 8 encontra-se na classificação das despesas de recursos individuais em função da sua correlação com mudanças na produção (geralmente atividades). Cada despesa de recursos é classificada como variável ou fixa, dependendo do quanto é ajustável dentro de um horizonte de tempo específico por causa das mudanças incrementais no volume de demanda das quantidades de saída. Essa abordagem para definir o comportamento do custo visando fornecer informações incrementais, representa uma melhoria dos níveis anteriores. Um exemplo de uma abordagem não-ABC, que tem desenvolvido capacidades de previsão sofisticadas é o método alemão de contabilidade de custos chamado Grenzplankostenrechnung, abreviado como GPK.

As organizações que planejam no nível 9b já alcançaram o nível 9a. As organizações no nível 9b determinam o impacto esperado sobre os recursos de todos os pedidos previstos do cliente ou sobre alterações do processo. As organizações no nível $9 \mathrm{~b}$ alavancam as relações de consumo para calcular as necessidades de capacidade de recursos. As organizações do nível $9 \mathrm{~b}$ primeiro comparam as necessidades previstas para os tipos de capacidade de recursos e para as quantidades já existentes. Elas, então, determinam se os tipos e as quantidades de recursos devem ser adicionados ou excluídos.

\section{Nível 10 - Time-driven ABC - TDABC}

As organizações no nível 10 utilizam exclusivamente os condutores de atividade baseados no tempo como padrão. Em contraste com o "empurrar" (de baixo para cima) o ABRP baseado no ABC do nível 9, onde as taxas de consumo de atividade são recalibradas depois de determinado o custo dos recursos do período anterior. O TDABC do nível 10 equipara taxa de tempo de cada atividade de saída como uma taxa normal, e calcula cada custo de atividade como um "custo padrão". Como todos os custos de atividade TDABC são calculados "no custo padrão", então a diferença líquida entre os custos dos recursos atuais e o custo da atividade padrão agregada pode ser calculada. Essa diferença pode ser um substituto para a capacidade não utilizada ou deficiente.

Nos casos em que a produção real é maior do que a prevista, a variância da capacidade excedente / ociosa será calculada como um número negativo. O TDABC do nível 10 se concentra principalmente nos custos diretos (como no nível 5). É, portanto, mais aplicável a organizações com eventos bastante repetitivos que são caracterizados por conexões relativamente menos indiretas - diretas em relação a organizações com custos significativamente indiretos. 
Nível 11 - Resource Consumption Accounting - RCA

As organizações que planejam no nível 11 são semelhantes as do nível 10, as quais também atingiram o nível 9, mas o nível 11 substitui as relações atividade-para-atividade para um diagrama de rede dos custos em múltiplos estágios com relações recurso-para-recurso ou atividade-para-recurso, onde a compreensão destas relações é fundamental para refletir adequadamente os custos dos recursos e suas características.

A capacidade encontra-se fisicamente em um recurso, e não em atividades ou produtos finais. Os recursos são o ponto de partida para o gerenciamento de capacidade e custos. O nível 11 fornece informações altamente divisíveis do custo marginal e do custeio por absorção em todos os objetos de custo, que são compilados com uma simples ênfase a aderir ao princípio de causalidade. Semelhante ao nível 10, o nível 11 determina o impacto esperado sobre todos os recursos de todas as previsões de pedidos do cliente ou de alterações do processo.

Em resumo, as organizações do nível 11 integram tanto o feedback do controle descritivo dos custos para ações corretivas quanto o planejamento preventivo dos recursos em um único modelo. Este é o nível de alcance do Resource Consumption Accounting. Destaca-se que o RCA é um abrangente sistema de gestão, totalmente integrado de custos, que fornece aos gestores informações otimizadas sobre a organização, uma vez que combina a aprendizagem, aplicabilidade e princípios para tomada de decisões, permitindo uma adequada e eficiente gestão do negócio. O RCA é um instrumento válido para o processo de planejamento e controle em uma empresa.

\section{Nível 12 - Simulação (Simulation)}

As organizações que planejam no nível 12 têm indiscutivelmente atingido o nível mais alto de planejamento de custos. A simulação pode acontecer de diversas formas e graus de sofisticação. Um exemplo é a chamada "dinâmica de sistemas", que, em termos simples, reflete as interdependências simultâneas dos fatores e influências em qualquer entidade ou organização. A simulação é uma forma elevada de modelagem, onde esta representa operações físicas.

As simulações do nível 12 abrangem a capacidade de planejamento futuro, um termo utilizado por planejadores de produção que projetam e programam a produção de um mix diversificado de produtos, utilizando um vasto conjunto de equipamentos diferenciados. Esse tipo de planejamento simulado inclui a realidade do processamento e os tempos de espera, as posições dos produtos, taxas de consumo da capacidade de recursos e as limitações de capacidade.

\section{CONSIDERAÇÕES FINAIS}

O artigo buscou explorar a conceituação, operacionalização, as aplicações e limitações da metodologia do Resource Consumption Accounting (RCA) ou Contabilidade de Consumo de Recursos, que constitui uma nova abordagem da gestão estratégica de custos e que fornece aos gestores informações otimizadas sobre a organização. Buscou-se através desta pesquisa apresentar uma metodologia para aplicação do RCA, que é a análise dos níveis de maturidade dos custos. Destaca-se que, justamente os níveis de 9 a 12, que alteram do ponto de vista descritivo da medição de custos para o ponto de vista preditivo da economia gerencial para a tomada de decisão. Esses quatro níveis influenciam as informações dos níveis 6 
a 8 para projetar os recursos necessários (e seus custos associados), de modo a coincidir com as quantidades de demanda previstas com capacidade ofertada.

Os níveis de 9 a 12 equilibram a ênfase no custo do produto final com recursos consumidos por ele. De certa forma, esses quatro níveis revertem o fluxo do Custo Contínuo dos níveis 1 a 8 , onde o foco era a saída resultante do consumo de atividades de trabalho. Do nível 9 ao nível 12 as capacidades de previsão exigem a habilidade para executar o modelo de trás para frente (partindo do custo final do produto para os recursos). O nível 11 é a abordagem do Resource Consumption Accounting ou da contabilidade do consumo de recursos (RCA). O RCA realça os recursos e as características de seu custo como o ponto de partida para todos os fluxos de custos através do modelo. Essencialmente, o RCA combina a aprendizagem, aplicabilidade e princípios para tomada de decisões, permitindo uma adequada e eficiente gestão do negócio.

Apesar de toda ênfase da literatura quanto às necessidades de adoção das práticas de gestão estratégica de custos, os achados desta pesquisa buscaram corroborar trabalhos sobre o tema do RCA, mesmo que com uma literatura referencial apenas estrangeira e bastante escassa. Comparativamente, aos conceitos da literatura mais recente sobre gestão de custos, parece que ainda há muito a evoluir na sua utilização pelas empresas, especialmente brasileiras. É importante que cada vez mais acadêmicos e pesquisadores busquem o aperfeiçoamento das práticas e métodos de gestão de custos de forma a viabilizar suas aplicações nas organizações, visando seu melhor desempenho.

\section{REFERÊNCIAS}

CHING, Hong Yuh. Gestão de estoques na cadeia de logística integrada - Supply Chain. 2. ed. São Paulo: Atlas, 2001. CLINTON, B. Douglas; WEBBER, Sally A. RCA at Cloplay. Strategic Finance. p. 21-26, out. 2004.

CREPALDI, Silvio A. Contabilidade gerencial: teoria e prática. 4 ed. São Paulo: Atlas, 2009.

FRIEDL, Gunther; HANS-ULRICH, Kupper; BURKHARD, Pedell. Relevance Added: Combining ABC with German Cost Accounting. Strategic Finance. p.56-61, jun. 2005.

GIL, Antônio C. Métodos e técnicas de pesquisa social. 5 ed. São Paulo: Atlas, 1999.

GRASSO, L. P. Are ABC and RCA Accounting Systems Compatible with Lean Management? Management Accounting Quarterly, v. 7, n. 1, p.12-27, 2005.

HANSEN, Don R.; MOWEN, Maryanne M. Gestão de custos: contabilidade e controle. São Paulo: Pioneira Thomson Learning, 2001

IFAC PAIB Committee International Good Practice Guidance, 2011. Disponível em: http://www.ifac.org/Members/DownLoads/evaluating-and-improving-co.pdf . Acesso em: 10 mai. 2016.

IFAC PAIB: A Costing Levels Continuum maturity Model, 2011. Disponível em http://www.ifac.org/Members/DownLoads/evaluating-the-costing-jour.pdf. Acesso em 06 abr. 2011.

KAPLAN, Robert S.; ANDERSON, Steven.R.: Time-Driven Activity-Based Consting - A simpler and more powerful path to higher profits. Boston: Harvard Business School Press, 2007.

KRUMWIEDE, Kip R. Rewards And Realities of German Cost Accounting. Strategic Finance. p.27-34, abr. 2005.

LAKATOS, Eva M.; MARCONI, Marina de A.. Fundamentos de metodologia científica. 5 ed. São Paulo: Atlas, 2002.

MARTINS, Eliseu. Contabilidade de custos. 9 ed. São Paulo: Atlas, 2003.

MERWE, Anton van der; KEYES, David E. The case for resource consumption accounting. Strategic Finance. p. 1-6, abr. 2002.

POMPERMAYER, Cleonice B.; LIMA, João E. Gestão de custos. Gazeta do Povo. (Coleção Gestão Empresarial - FAE). Curitiba, v. 4, 50p. 2002. RCA Institute: http://www.rcainstitute.org/rcai-3-WhatlsRCA.php . Acesso em: 10 abr. 2016.

RICHARDSON, Robert Jarry. Pesquisa social: métodos e técnicas. São Paulo: Atlas, 1999. 
SCHMIDT, Paulo; SANTOS, José L. ; LEAL, Ricardo. Time-driven activity based costing (TDABC): uma ferramenta evolutiva na gestão de atividades. In: Congreso Iberoamericano de Contabilidad de Gestión, 3, 2009, Valencia, Espanha.

SHARMAN, Paul A.; KURT Vikas. Lessons from German Cost Accounting. Strategic Finance. p. 28-35, dec. 2004. SHARMAN, Paul A. Bring On German Cost Accounting. Strategic Finance, p.2-9, dec- 2003.

SOUZA, Marcos A.; SILVA, Élio J.; PILZ, Nestor. Estrutura e práticas de gestão estratégica de custos: um estudo em uma empresa multinacional brasileira. In: Congresso USP FIPECAFI 2010, São Paulo. Disponível em:

<http://www.congressousp.fipecafi.org/artigos102010/242.pdf>. Acesso em: 03 abr. 2016. 